\title{
R (Transmissible Drug-resistance) Factors in Salmonella typhimurium: Pattern of Transduction by Phage P 22 and Ultraviolet-protection Effect
}

\author{
By W. T. DRABBLE* AND B. A. D. STOCKER \\ Guinness-Lister Research Unit, Lister Institute of Preventive Medicine, \\ Chelsea Bridge Road, London, S.W. I
}

(Accepted for publication 2I March I968)

SUMMARY

$\mathrm{R}$ factors from Enterobacter strains isolated outside Japan were transferred to Salmonella typhimurium LT 2 and their resistance traits transduced by phage $\mathrm{P} 22$. Of five $f^{+}$factors conferring resistance to tetracycline (Tc), streptomycin $(\mathrm{Sm})$, sulphonamides $(\mathrm{Su})$ and chloramphenicol $(\mathrm{Cm})$, three, one of which conferred also resistance to kanamycin $(\mathrm{Km})$, behaved like previously reported factors in that: Tc was transduced by itself but never with any other trait; all other traits were usually co-transduced; no transductants could transmit resistance by conjugation, even after infection with $\mathrm{F}^{\prime}$-I3 lac. The other two $\mathrm{fi}^{+}$factors, which conferred also resistance to benzylpenicillin (Pn), behaved similarly, except that: Pn was transduced only at a low rate, and was never co-transduced with any other trait; and a few transductants when given $\mathrm{F}^{\prime}-\mathrm{I} 3$ lac became able to transmit. Four $f^{-}$ factors, two of type Tc, Sm, Su, Pn and two of type Tc, Su, Pn differed in that: nearly all Tc transductants acquired also all the other traits; Pn was usually co-transduced with the other traits; and some transductants could transmit by conjugation, even without $\mathrm{F}^{\prime}$-I3 lac. The various transductant classes obtained by treatment with $\mathrm{P} 22$ grown on LT 2 carrying both an $f^{+}$(Tc, Sm, $\mathrm{Su}, \mathrm{Cm}, \mathrm{Km}$ ) and $f^{-}$(Tc, Sm, Su, Pn) factor could be accounted for by transduction of fragments of either one or the other of these factors. The sorts of transductants produced by lysates of a strain possessing $\mathrm{Tc}, \mathrm{Sm}, \mathrm{Su}$, $\mathrm{Cm}, \mathrm{Km}$ determinants obtained by growing a strain carrying an $\mathrm{fi}^{+}(\mathrm{Tc}, \mathrm{Sm}$, $\mathrm{Su}, \mathrm{Cm})$ factor with one carrying an $f^{+}(\mathrm{Km})$ factor suggested that the strain carried two $f^{+}$factors, not a recombinant factor. Both $f^{-}$and $f^{+}$factors conferring resistance to benzylpenicillin conferred also resistance to cephalothin and cephaloridine and caused the constitutive production of a $\beta$-lactamase, active on both benzylpenicillin and on cephalothin. All four $\mathrm{fi}^{-}$and one of the five $f^{+}$factors resembled colI factors in that they protected strain LT 2 against the bactericidal effect of ultraviolet irradiation.

\section{INTRODUCTION}

Transmissible resistance to several antibiotics and to sulphonamides was encountered in Shigella strains in Japan in 1955, and soon became very common in that country (Watanabe, 1963; Mitsuhashi, 1965). Such resistance results from the presence in the bacteria of a non-chromosomal genetic determinant or plasmid, termed an $\mathbf{R}$ factor, composed of DNA of a characteristic base composition (Falkow, Citarella, Wohlhieter \& Watanabe, 1966). R factors reported from Japan commonly confer

* Present address: Department of Physiology and Biochemistry, University of Southampton, Southampton, $\mathrm{SO} 95 \mathrm{NH}$. 
four resistance traits, namely, resistance to tetracycline (Tc), streptomycin $(\mathrm{Sm})$, sulphonamides $(\mathrm{Su})$ and chloramphenicol $(\mathrm{Cm})$; less often, only one, two or three of these traits. Similar R factors are now prevalent in many parts of the world and factors determining resistance to the neomycin-kanamycin (Km) group (Lebek, I963; Carpenter \& Drabble, 1965) or to the penicillins (Anderson \& Datta, 1965; Carpenter \& Drabble, 1965) have been encountered. Some $\mathrm{R}$ factors, termed $\mathrm{fi}^{+}$, when introduced into Hfr or $\mathrm{F}^{+}$lines of Escherichia coli decrease or abolish their fertility and susceptibility to the male-specific phages, probably by repressing formation of F-pili (Watanabe et al. 1964a; Meynell \& Datta, 1965; Datta, Lawn \& Meynell, I966). The $f$ property divides $\mathrm{R}$ factors into two groups. The presence in a host of an $\mathrm{R}$ factor of one group interferes with its acquisition and maintenance of another factor of the same group, but not with that of a factor of the other group (Watanabe et al. 1964a). $\mathrm{R}$ factors or their component genes may be transduced by general transducing phages. The Escherichia coli phage P I commonly transduces an entire R factor, i.e. most transductants acquire all the drug-resistance traits conferred by the factor, and also the ability to transmit these traits by conjugation. In Salmonella, by contrast, the general transducing phages, $\mathrm{P} 22$ for group $\mathrm{B}$ and $\epsilon_{15}$ and $\epsilon_{34}$ for group $\mathrm{E}$, transduce only fragments of the $\mathrm{R}$ factor. The Tc trait when present is transduced separately from the other traits, which usually travel together; and the transductants cannot transmit their resistance traits by conjugation-presumably because the transduced fragments of the $\mathrm{R}$ factor lack its genes which confer ability to conjugate (Watanabe \& Fukusawa, I96r; Harada, Kameda, Suzuki \& Mitsuhashi, I963). Most isolates of another sort of plasmid, namely, colicine factors of the colIa and colIb classes, decrease the sensitivity of their bacterial host to the bactericidal action of ultraviolet irradiation (Howarth, 1965; Howarth-Thompson, 1966) and we have found (Drabble \& Stocker, 1966) that some $\mathrm{R}$ factors have a similar effect in Salmonella typhimurium strain LT 2. We here report a comparative study of the transduction and u.v.-protecting properties of various $\mathrm{R}$ factors. They include factors conferring penicillin-resistance, discovered in S. typhimurium strains isolated in England (Anderson \& Datta, 1965); the $\mathrm{R}$ factor conferring resistance to kanamycin (Lebek 1963); and several $\mathrm{R}$ factors found amongst Shigella strains from various parts of the world examined at the Shigella Reference Laboratory, Central Public Health Laboratory, Colindale, London, N.W. 9. (Carpenter \& Drabble, 1965).

\section{METHODS}

Bacterial strains and phages. The strains from which the $\mathrm{R}$ factors were obtained are listed in Table I. Strains with sL numbers were from the collection maintained in this laboratory. The Escherichia coli strains W I 895 and $200 \mathrm{U}$ were provided by Dr P. Gemski and came originally from J. Lederberg and F. Jacob respectively. Phage P 22 was used for transduction. The male-specific phage $\mathrm{R}_{17}$ (Loeb, 1960) was used to test the $f i$ character of $\mathbf{R}$ factors.

Media. Nutrient broth was Oxoid no. 2, code CM 67. Nutrient agar was Oxoid blood agar base (code CM 55). For some purposes peptone agar (Evans's bacteriological peptone, I \%,w/v) was used instead. The defined medium was that of Davis $\&$ Mingioli (1950) with glucose, I mg./ml., solidified with Oxoid no. 3 agar at 15 g./1. Appropriate amino acids and purines were added at $10 \mu \mathrm{g} . / \mathrm{ml}$.; vitamins at $\mathrm{I} \mu \mathrm{g} . / \mathrm{ml}$. Antibacterial drugs. Bacterial sensitivity was tested by inoculation on nutrient agar 


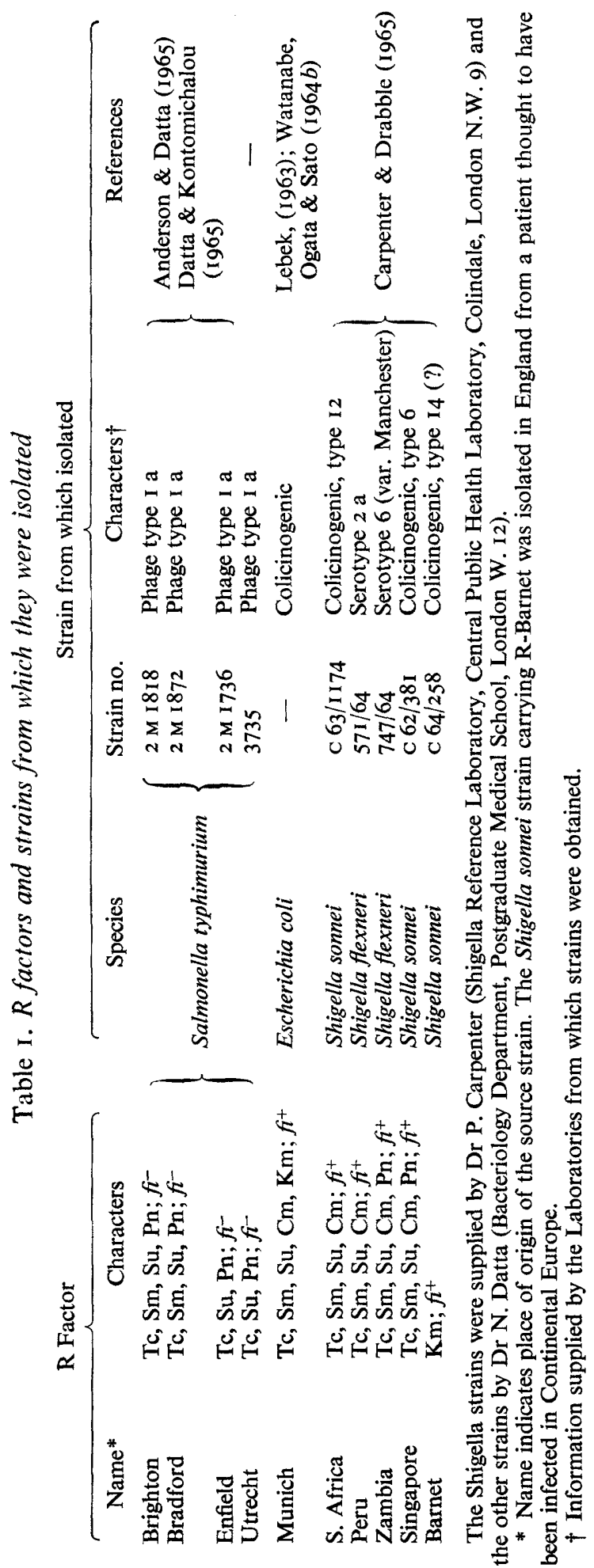


or peptone agar containing the following: tetracycline, $25 \mu \mathrm{g} . / \mathrm{ml}$.; streptomycin sulphate, either $25 \mu \mathrm{g} . / \mathrm{ml}$, , to test the low-level resistance conferred by some of the $\mathrm{R}$ factors, or $\mathrm{I} \mathrm{mg} . / \mathrm{ml}$., to test the high-level (mutational) resistance of some strains; chloramphenicol, $25 \mu \mathrm{g} . / \mathrm{ml}$.; benzylpenicillin, $60 \mu \mathrm{g} . / \mathrm{ml}$.; kanamycin, $25 \mu \mathrm{g} . / \mathrm{ml}$. Sulphonamide-sensitivity was determined by inoculation to defined medium containing sulphathiazole, I mg. $/ \mathrm{ml}$. The drug-resistance pattern of sets of 25 transductants, etc., and of two control strains was determined by replication with a 27-prong replicator to test plates of the above media. Nutritional characters and sugar fermentation characters were similarly determined by replication to appropriate media.

Conjugational transfer of plasmids. $\mathrm{R}$ factors and the $\mathrm{F}^{\prime}$ - $\mathrm{I} 3$ lac episome were transferred from appropriate donor strains to recipients by growing them together for about $18 \mathrm{hr}$ in broth at $37^{\circ}$ without shaking or aeration, then plating on medium selective for bacteria of the recipient strain which had received one of the characters of the plamid. The R factors originally harboured in Salmonella typhimurium strains, all of which conferred penicillin resistance, were first transferred in this way to SL 675 (LT 2 pur pro str), selection being for penicillin resistance and for the high-level streptomycin resistance of the recipient. The R-Munich factor was transferred directly from its original Escherichia coli host to SL 726 (LT 2 met trp ara gal), using medium containing kanamycin with citrate as sole energy source to select the Salmonella recipient. Attempts to transfer $\mathbf{R}$ factors directly from their original Shigella hosts to SL 726 were unsuccessful. They were therefore first transferred to an $E$. coli $\mathrm{K} 12$ met $\mathrm{F}^{-}$strain, selecting for nicotinic-acid independence and for one of the drugresistance traits conferred by the $\mathrm{R}$ factor. Each of these $\mathrm{R}$ factors was then easily transferred from the $E$. coli to SL 675 , selection being made for the high-level streptomycin resistance of the acceptor as well as an R-factor-determined resistance trait. The $\mathrm{R}$ factors thus introduced into the streptomycin-resistant LT 2 line, SL 675, were next transferred to a streptomycin-sensitive LT 2 line, SL 726 (LT 2 met trp ara gal) with selection for the $\mathrm{pur}^{+} \mathrm{pro}^{+}$of the recipient and for one of the resistance traits of the $\mathrm{R}$ factor.

The $f i$ character of the $\mathrm{R}$ factors was determined after transfer from LT 2 to an Escherichia coli K I2 met Hfr line, W I 895. To test for inhibition of fertility the R derivatives of $\mathrm{W} I 895$ were scored for ability to produce $l a c^{+}$str-r recombinants when crossed to a $\mathrm{K}$ I 2 lac str- $r \mathrm{~F}^{-}$line, $200 \mathrm{U}$. The $\mathrm{W}$ I 895 lines given $\mathrm{R}$ factors were also tested for sensitivity to the male-specific phage $\mathrm{R} \mathrm{I} 7$ (on tryptone yeast-extract agar containing $0.002 \mathrm{M}-\mathrm{CaCl}_{2}$ ).

Transduction. Phage $\mathrm{P} 22$ was propagated by the soft-agar layer method on the SL 726 (LT 2 met trp ara gal) derivatives carrying the various $\mathrm{R}$ factors. The lysates after centrifugation were Seitz-filtered or held at $60^{\circ}$ for $\mathrm{r}$ hr. The strain used as transductional recipient was SL 862 (LT 2 trp his). A young broth culture was mixed with phage at a multiplicity of about 10. After $10 \mathrm{~min}$. the mixture was centrifuged and the bacteria washed twice, then resuspended at $\times 20$ the original concentration in $1 / 4$ strength Ringer solution. Drug-resistant transductants were selected by spreading $0.1 \mathrm{ml}$. volumes of the concentrated suspension on duplicate plates of peptone agar containing a single antibiotic, or of supplemented defined medium with sulphathiazole. As controls, bacteria to which no phage had been added were similarly washed and plated. Suitable dilutions of the transduction mixtures were also plated on defined medium supplemented with tryptophan, to detect the rate of transduction of his ${ }^{+}$. 
The drug-resistance pattern of transductants was determined, after purification, as described above. The ability of representative drug-resistant transductants to transmit their resistance traits by conjugation was tested by overnight growth in broth with strain RS 75, a streptomycin-resistant LT 2 line resistant to P 22 (and in consequence unsusceptible to transduction) (Gemski \& Stocker, 1967). Drops from overnight mixed cultures of each transductant with RS 75 were spotted on drug-supplemented peptone agar selective for the high-level streptomycin-resistance of the acceptor and one of the resistance traits of the transductant. Transductants found unable to transmit were infected with the $\mathrm{F}^{\prime}-\mathrm{I} 3$ lac episome by growth with Salmonella typhimurium strain SL 2 I IO (leu cys mal rou $\mathrm{F}^{\prime}$-I 3 lac), followed by selection on appropriate medium. The $\mathrm{lac}^{+}$derivatives were then tested for ability to transmit drug-resistance, as before.

Test for protection against u.v. radiation. The effect of $\mathrm{R}$ factors on susceptibility to killing by ultraviolet irradiation was tested in Salmonella typhimurium SL 675 (pur pro str). Overnight $37^{\circ}$ broth cultures were diluted I/Io in broth; the diluted cultures were incubated at $37^{\circ}$, without aeration or shaking, for $4-5 \mathrm{hr}$, by which time they contained $3-5 \times 10^{8}$ viable bacteria $/ \mathrm{ml}$. Serial decimal dilutions were made in quarterstrength Ringer solution containing $10 \%(\mathrm{v} / \mathrm{v})$ nutrient broth. Drops (volume $0.02 \mathrm{ml}$.) from appropriate dilutions were delivered to the surface of peptone agar plates. When the drops had dried, sets of plates were exposed for various times, usually $30 \mathrm{sec}$. and $60 \mathrm{sec}$, at $69.5 \mathrm{~cm}$. from a $15 \mathrm{~W}$ Phillips TUV Germicidal Lamp (low-pressure $\mathrm{Hg}$ arc). Colonies on the irradiated, and unirradiated control, plates were counted after overnight incubation at $30^{\circ}$. The medium and temperature of incubation were chosen to give conveniently small colonies.

Table 2. Maximum antibiotic concentrations $(\mu g . / \mathrm{ml}$.) allowing growth of strain SL 726 and of its derivatives carrying R-Munich or R-Brighton

$\begin{array}{lccc} & \text { SL } 726 & \text { SL 726 } & \text { SL 726 } \\ \text { Tetracycline } & 0 \cdot 5 & 60 & 50 \\ \text { Streptomycin } & \text { I } & 20 & 60 \\ \text { Sulphathiazole } & 40 & 2500 & 2500 \\ \text { Chloramphenicol } & 2 \cdot 5 & 1000 & 2 \cdot 5 \\ \text { Kanamycin } & 0 \cdot 5 & 1000 & 0 \cdot 5 \\ \text { Neomycin } & 0 \cdot 5 & 100 / 150 & 0 \cdot 5 \\ \text { Benzylpenicillin } & \mathrm{I} \cdot 5 & \mathrm{I} \cdot 5 & 360 \\ \text { Cephalothin } & \mathrm{I} \cdot 5 & \mathrm{I} \cdot 5 & 100 \\ \text { Cephaloridine } & 1 \cdot 25 & \mathrm{I} \cdot 25 & 35\end{array}$

Inocula of about $1 \mathrm{O}^{4} \mathrm{log}$-phase cells were added to $3 \mathrm{ml}$. tubes of nutrient broth containing graded concentrations of drug. Growth was inferred from turbidity after $20 \mathrm{hr}$ at $37^{\circ}$.

\section{RESULTS}

\section{Pattern of resistance conferred by $R$ factors}

Table 2 records the minimum concentration of various drugs required to inhibit the growth of a small inoculum of a Salmonella typhimurium LT 2 line carrying no $\mathrm{R}$ factor, and of its derivatives carrying R-Munich (Tc, Sm, Su, Cm, Km) or R-Brighton (Tc, Sm, Su, Pn). When the R factor conferred resistance, the minimal inhibitory concentration was increased at least 20 -fold. Several $\mathrm{R}$ factors conferring resistance to benzylpenicillin, derived either from $S$. typhimurium strains or from Shigella strains, 
conferred also substantial resistance to 6-amino penicillanic acid, cloxacillin, phenethicillin, methicillin, cephalothin and cephaloridine. Salmonella typhimurium and Escherichia coli lines given the complete R-Brighton factor by conjugation or transduction and an LT 2 line given only its benzylpenicillin-resistance trait by transduction gave positive reactions in the test of Foley \& Perret (1962), indicating production of a $\beta$-lactamase, active on both benzylpenicillin and also, though less strongly, on cephalothin. A strain of Bacillus cereus with an inducible penicillinase, included as control, gave as expected a positive reaction only with benzylpenicillin. Prior exposure of the colonies to chloroform vapour did not diminish the size of the zones produced in the penicillinase test, except in the case of the inducible $B$. cereus strain. That is, the $\mathrm{R}$ factors caused constitutive production of penicillinase. However, the chloroformexposed colonies produced zones more slowly than the untreated colonies in the test for cephalothin destruction.

\section{Conjugal transfer of $R$ factors}

All the $\mathrm{R}$ factors were transferred from their original hosts to the Salmonella typhimurium LT 2 line SL 726, as described under Methods. In each instance it was easy to isolate an LT 2 derivative with all the resistance traits of the original strain. This suggests that the complete R factor was being transferred. The Escherichia coli strain and several of the Shigella strains used as sources of $\mathbf{R}$ factors were colicinogenic, but the LT 2 derivatives chosen for investigation had not acquired colicinogeny with the $\mathrm{R}$ factor. Some R factors are very unstable in S. typhimurium LT 2 (Watanabe, I963). In our experiments spontaneous loss of resistance traits was not so frequent as to interfere with experimental design. To investigate its stability, the LT 2 (R-Munich) line, resistant to tetracycline, streptomycin, sulphonamide, chloramphenicol and kanamycin, was subcultured in broth daily. After five subcultures $50 \%$, and after 13 subcultures $99 \%$, of the population had lost resistance to all drugs except tetracycline. After I6 subcultures $40 \%$ of the population was no longer tetracycline-resistant, and $99 \%$ no longer resistant to the other drugs.

\section{Strains carrying two $R$ factors}

Strains presumably carrying both an $f^{-}$and an $f^{+} \mathrm{R}$ factor were isolated from mixed cultures of derivatives of the Salmonella typhimurium strains SL 699 (met trp) and SL 726 (met trp ara). One strain carried R-Munich (Tc, Sm, Su, Cm, Km) and the other R-Brighton (Tc, Sm, Su, Pn). Doubly resistant colonies were selected on peptone arabinose indicator agar containing $\mathrm{Pn}$ and $\mathrm{Km}$. The medium distinguished the arabinose-fermenting SL 699 from the arabinose-negative SL 726 line. Whichever strain acted as donor the fraction of bacteria carrying R-Munich which acquired R-Brighton was about $1 \mathrm{O}^{-3}$, and the fraction of bacteria carrying $\mathrm{R}$-Brighton which acquired R-Munich was about $2 \times 10^{-5}$. The colonies selected carried all the resistance traits of both donor and recipient, and were presumed to carry both factors. Strains apparently carrying two $f^{+} \mathrm{R}$ factors were prepared by growing $S$. typhimurium SL 726 (met trp gal) carrying either R-S. Africa (Tc, Sm, Su, Cm) or R-Peru (Tc, Sm, Su, Cm) with the Shigella strain $\mathrm{C} 64 / 25^{8}$, carrying R-Barnet $(\mathrm{Km})$. On plating the mixtures on peptone galactose indicator medium containing $\mathrm{Cm}$ and $\mathrm{Km}$, double-resistant galactosefermenting Shigella colonies were distinguishable from doubly resistant galactose-nonfermenting Salmonella colonies. The fraction of Shigella organisms carrying R-Barnet 
which acquired the episome from Salmonella was about $5 \times 10^{-7}$ for both R-S. Africa and R-Peru. For the reverse transfer, of R-Barnet from Shigella to Salmonella, the fraction was about $5 \times 10^{-7}$ when the recipient carried R-S. Africa, but 20-fold higher when the recipient carried R-Peru. All the colonies isolated in this way carried all the unselected resistance traits of the original strains. The Salmonella strains presumably carrying two $f^{+} \mathrm{R}$-factors appeared quite stable and could be used in transductional studies with phage $\mathrm{P} 22$.

\section{Transduction of $R$ factor resistance traits by phage $P 22$}

Yield of transductants. No colonies, or negligibly few, appeared on the control plates, except when selection was made for low-level streptomycin-resistance, when they were usually so numerous as to obscure possible transductant colonies on the experimental plates. In some experiments, however, transductants recognizable because of having unselected donor resistance traits were obtained, together with mutants, by selection for low-level streptomycin-resistance. The numbers of transductant colonies obtained by selection for various resistance traits are recorded in

Table 3. Yield of drug-resistant and of his transductants after treatment with phage $P 22$ grown on donor strain carrying various $R$ factors

(Donor strain was SL $726(=$ LT 2 met $A \operatorname{trp} B)$ carrying the indicated $\mathrm{R}$ factor. Recipient strain was SL $862(=$ LT 2 hisD $\operatorname{trp} A)$. Phage was added at multiplicity about IO.

\begin{tabular}{|c|c|c|c|c|c|c|c|}
\hline \multirow{2}{*}{$\begin{array}{l}\mathrm{R} \text { factor(s) } \\
\text { carried by } \\
\text { donor }\end{array}$} & \multicolumn{7}{|c|}{ No. of colonies/plate* on medium selective for } \\
\hline & $h_{i s^{+}}$ & Tc & $\mathrm{Sm}$ & $\mathrm{Su}$ & $\mathrm{Cm}$ & Pn & $\mathrm{Km}$ \\
\hline R-Munich & 336 & 14 & b.g.† & n.d. & 25 & $-\S$ & 13 \\
\hline R-S. Africa & 87 & I I & b.g. & n.d. & 70 & - & - \\
\hline R-Peru & 94 & 16 & b.g. & n.d. & 85 & - & - \\
\hline R-Brighton & 2 II & 132 & $>1000$ & 7 & - & $>1000$ & 一 \\
\hline R-Bradford & 280 & 98 & b.g. & 19 & - & 42 & - \\
\hline R-Enfield & 3 IO & 189 & - & 300 & - & 244 & - \\
\hline R-Singapore & 117 & 7 & b.g. & I & 70 & I3 & - \\
\hline R-Zambia & 128 & 4 & b.g. & 0 & 94 & I & - \\
\hline
\end{tabular}

* Plate inoculum about $10^{9}$ bacteria for drug-resistance selection, and about $10^{7}$ bacteria for selection of $h^{+}$.

$\dagger$ b.g. means that any transductant colonies were obscured by numerous resistant-mutant colonies, present also on control plate of bacteria without phage.

$\ddagger$ n.d. means this selection was not done.

$\S-$ means not relevant, since $\mathrm{R}$ factor of donor does not confer resistance to this agent.

Table 3, together with the frequency of is $^{+}$transduction, tested as a control. These figures, however, in some instances certainly underestimate the true number of transductants, for on occasion selection of resistance to one drug gave more transductants resistant to a second drug than were obtained by direct selection with the second drug. Also repeat experiments sometimes gave quite different numbers of colonies. For instance, in the experiment recorded in Table 3 the numbers of transductants obtained by selection for streptomycin-resistance or penicillin-resistance after treatment with phage grown on the donor carrying R-Brighton were exceptionally high (of the same order of magnitude as the number of his transductants)-but the frequency of unselected donor resistance traits confirmed that the clones were indeed transductants, not mutants. 


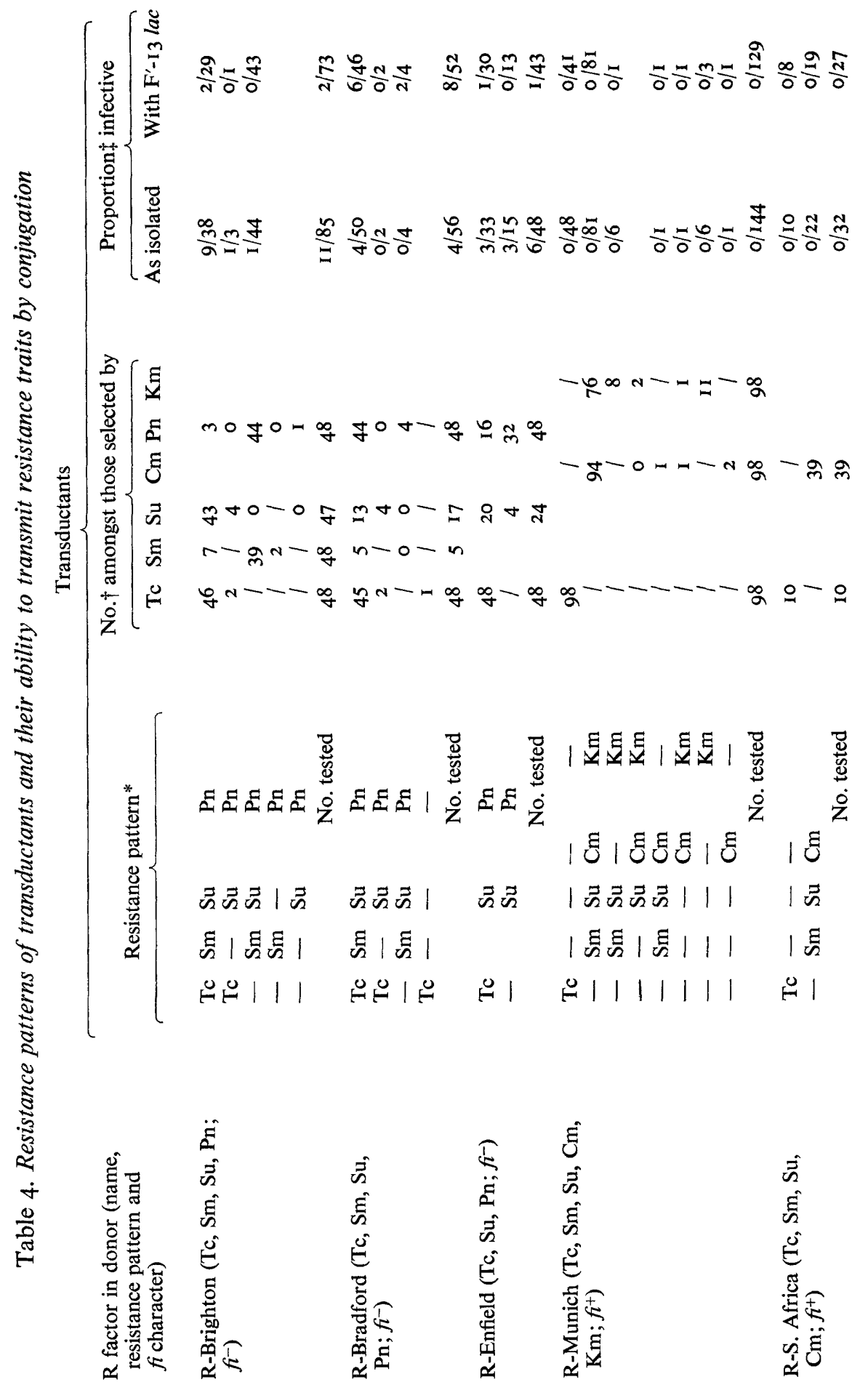




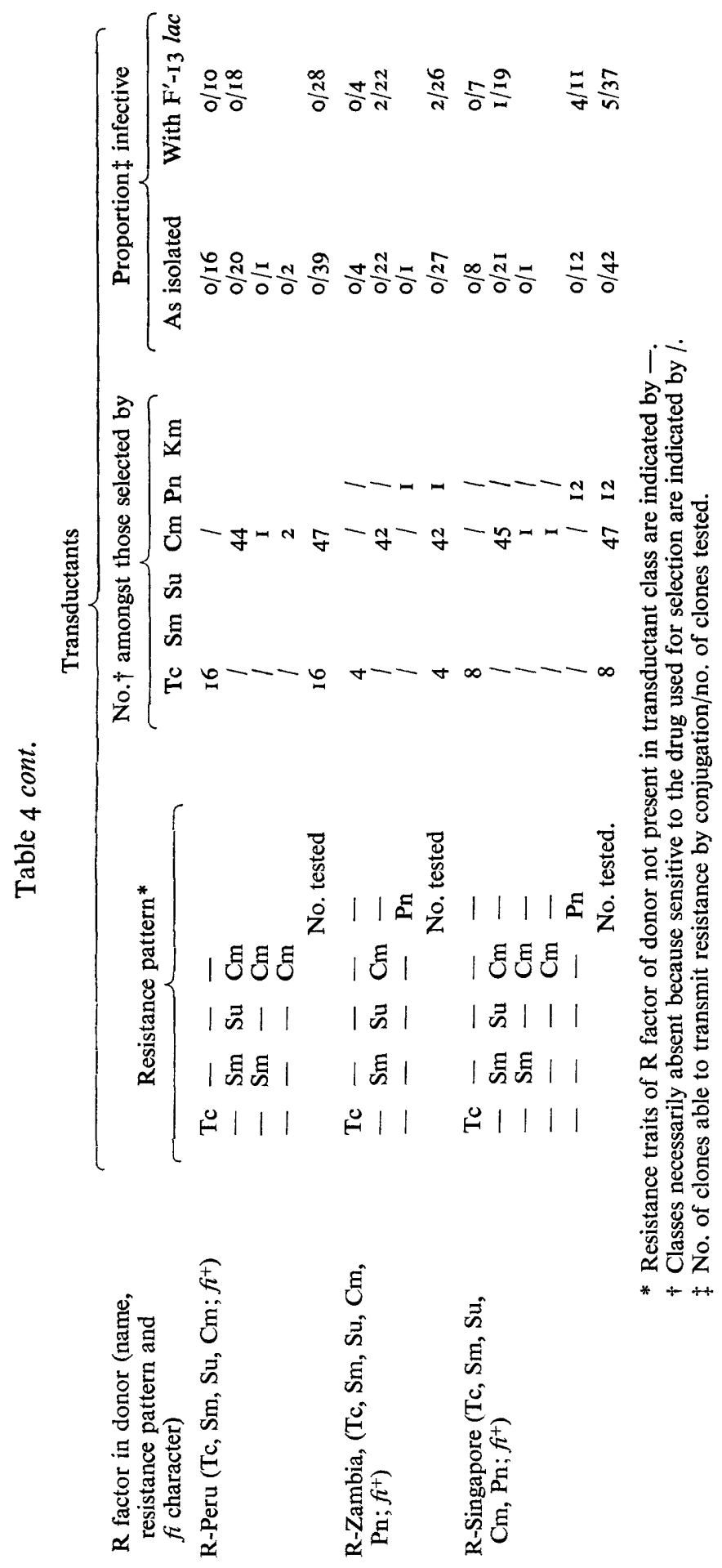


Resistance pattern of transductants. The resistance patterns of transductants selected by various drugs are recorded in Table 4 . In the case of the five $f^{+}$factors conferring multiple resistance, no transductant selected as tetracycline-resistant had any other resistance trait from the $\mathrm{R}$ factor present in the donor strain; and no transductant selected as chloramphenicol-resistant had the Tc trait of the R factor concerned, though most had its $\mathrm{Sm}$ and $\mathrm{Su}$ traits, and also its $\mathrm{Km}$ trait in the case of R-Munich. Most of the 98 transductants selected for acquisition of the $\mathrm{Km}$ trait of R-Munich had acquired also its $\mathrm{Sm}, \mathrm{Su}$ and $\mathrm{Cm}$ traits, but none had its Tc trait. Thus the $\mathrm{Km}$ determinant of this $\mathrm{fi}^{+}$factor is transduced by phage $\mathrm{P} 22$ as a member of the Sm, Su, Cm group-as reported by Watanabe et al. 1964b. Of the five $f^{+}$factors tested two, $\mathrm{R}$-Singapore and R-Zambia, conferred the Pn trait, in addition to the Tc, Sm, $\mathrm{Su}$ and $\mathrm{Cm}$ traits. The Pn trait was not present amongst $\mathrm{I} 2$ transductants selected by tetracycline (none of which showed any other resistance trait) nor amongst 89 selected by chloramphenicol (though nearly all of these had the unselected Sm and Su traits). Few colonies were obtained on selection for benzylpenicillin resistance (Table 3); no other resistance trait was present in the twelve transductants tested. It thus appears that in the $\mathrm{fi}^{+}$factors R-Singapore and R-Zambia the determinant of benzylpenicillinresistance is transduced by phage $\mathbf{P} 22$ separately from the Tc determinant and from the $\mathrm{Sm}, \mathrm{Su}, \mathrm{Cm}$ group.

Table 5. Resistance patterns of transductants derived from donors carrying two $R$ factors

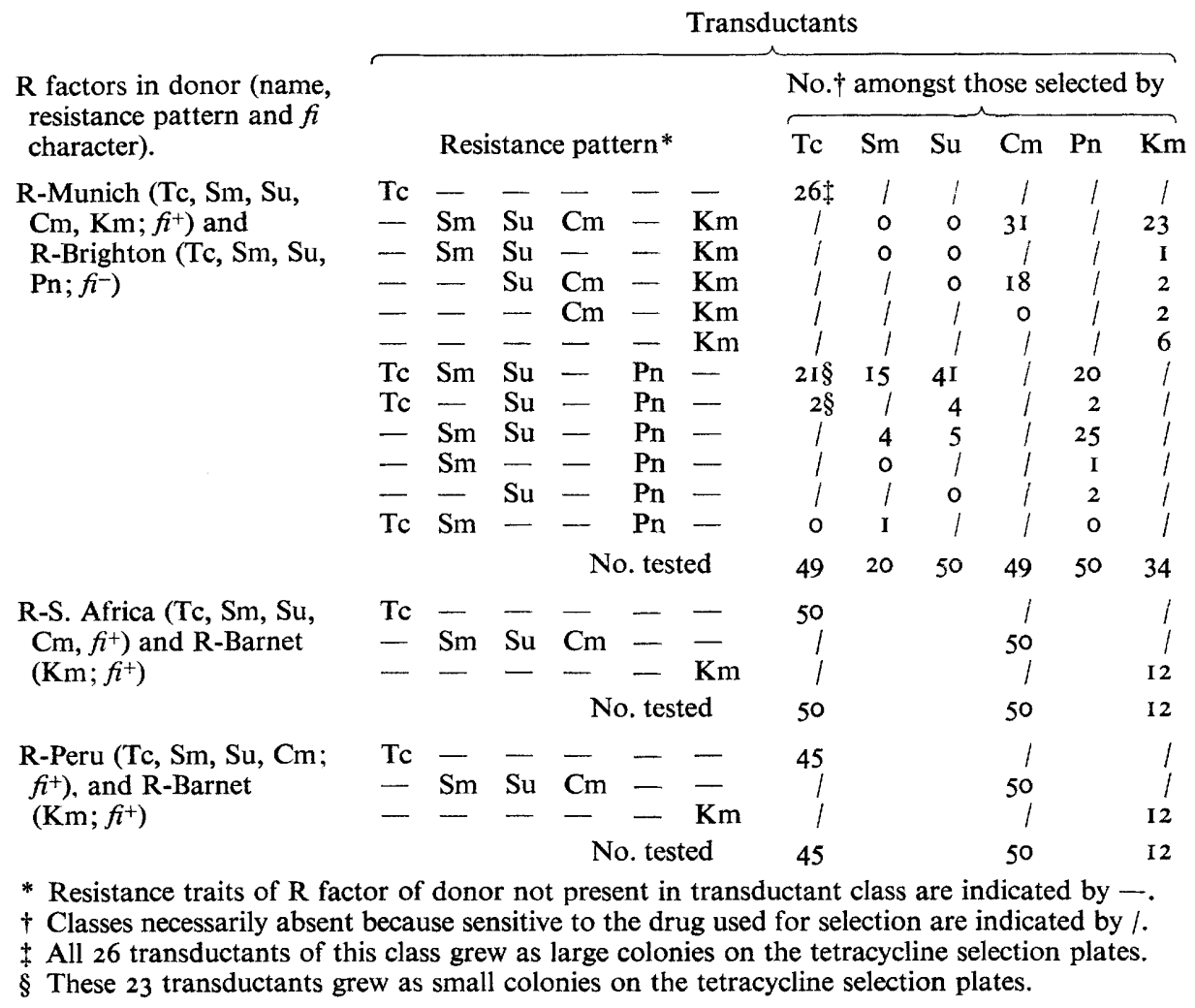


Three $f^{-}$factors, two of type Tc, Sm, Su, Pn and one of type Tc, Su, Pn and all derived from Salmonella typhimurium, were tested. In contrast with the results with $f^{+}$factors nearly all transductants selected by tetracycline had all the other resistance traits of the factor concerned, and many of those selected by streptomycin, sulphathiazole or benzylpenicillin had acquired tetracycline resistance (and other unselected resistance traits). Furthermore all of I44 transductants selected as benzylpenicillinresistant had acquired some or all of the other traits of the factor concerned. Most R-Utrecht transductants selected by tetracycline or by benzylpenicillin had all the three resistance traits of R-Utrecht. It thus appears that both the Tc and Pn determinants of these $f^{-} \mathbf{R}$ factors, unlike those of the $f^{+}$factors tested, can be transduced by phage $\mathbf{P} 22$ as part of a group comprising also the $\mathrm{Sm}$ and $\mathrm{Su}$ determinants.

Transduction from donors carrying two $R$ factors. When drug-resistant transductants were selected after treatment with phage $\mathbf{P} 22$ grown on donor strains carrying two $\mathrm{R}$ factors, R-Munich $\left(f^{+}, \mathrm{Tc}, \mathrm{Sm}, \mathrm{Su}, \mathrm{Cm}, \mathrm{Km}\right)$ and R-Brighton $\left(f i^{-}, \mathrm{Tc}, \mathrm{Sm}, \mathrm{Su}, \mathrm{Pn}\right)$, the classes obtained indicated independent transduction of the two $R$ factors-or rather parts of them. No transductant had two resistance traits, one derived from one factor and one from the other factor, and the different resistance patterns observed corresponded to transductant classes observed when the donor carried only one or the other of the two factors (Table 5). Two sizes of colony were observed when tetracyclineresistance was selected. All of 26 large colonies proved to be resistant to tetracycline only, and thus resembled the only class of tetracycline-resistant transductant obtained when the donor carried only the $f^{+}$R-Munich; all of 23 small colonies were resistant to several agents, including benzylpenicillin, and corresponded to the common classes of tetracycline-resistant transductant observed when the donor carried only the $f^{-}$ R-Brighton. Lysates of two strains each with the $\mathrm{Km}$ trait derived from the $f^{+}$ R-Barnet and the Tc, Sm, Su, $\mathrm{Cm}$ traits from another $f^{+}$factor produced both $\mathrm{Km}$ and $\mathrm{Sm}, \mathrm{Su}, \mathrm{Cm}$ transductants-but unlike the lysate of the strain carrying the $\mathrm{f}^{+}$ R-Munich factor ( $\mathrm{Tc}, \mathrm{Sm}, \mathrm{Su}, \mathrm{Cm}, \mathrm{Km}$ ) produced no transductants with both the $\mathrm{Km}$ trait and the $\mathrm{Sm}, \mathrm{Su}, \mathrm{Cm}$ traits together.

Ability of transductants to transmit resistance by conjugation. In general, Salmonella clones given R-factor drug-resistance traits by transduction are unable to transmit their resistance properties by conjugation. In testing for conjugation it is necessary to exclude the possible transfer by transduction due to phage liberated from lysogenic $\mathbf{R}^{+}$ bacteria, thus a strain unable to absorb P 22 was used as recipient. None of the 300 transductants of various classes derived from the five $f^{+} \mathrm{R}$ factors transmit by conjugation. By contrast a minority of the transductant clones with resistance traits derived from each of the three $f^{-} \mathbf{R}$ factors gave a positive result. The transductants able to transmit were of several different resistance patterns (Table 4). Several of them were found to transmit all their resistance traits simultaneously. It was reported by Harada, Kameda, Suzuki \& Mitsuhashi (1964) that some Salmonella transductants acquired the ability to transmit their resistance by conjugation after infection with $F^{\prime}-13$ lac. We transferred this factor to many of our non-transmitting transductants, and of $247 \mathrm{fi}^{+} \mathrm{R}$-factor transductants, seven acquired the ability to transmit drug resistance. All these were derived from $\mathrm{R}$ factors conferring resistance to benzylpenicillin. Of $\mathrm{I} 68 \mathrm{f}^{-}$R-factor transductants negative in the first test, II transmitted resistance by conjugation when they also carried $\mathrm{F}^{\prime}$-13 lac (Table 4). In the few tests made, the majority of acceptor colonies selected for acquisition of any one drug- 
resistance trait were found to have acquired all the resistance traits of the donor, and usually also its $l a c^{+}$property.

\section{Effect of $R$ factors on ultraviolet susceptibility}

Each R factor except R-Barnet was tested for ability to protect Salmonella typhimurium against killing by ultraviolet irradiation. The clones tested had acquired by conjugation all the drug-resistance traits of the $\mathrm{R}$ factor concerned, except possibly

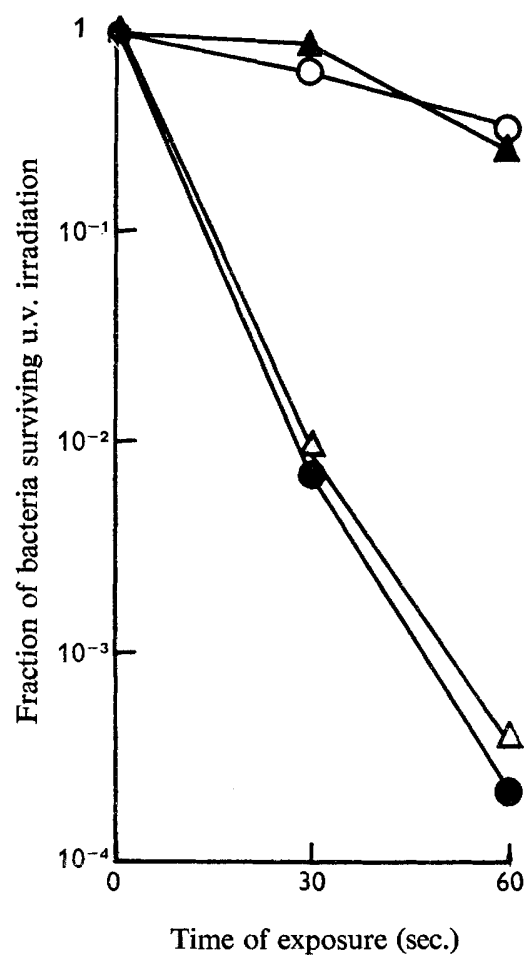

Fig. I

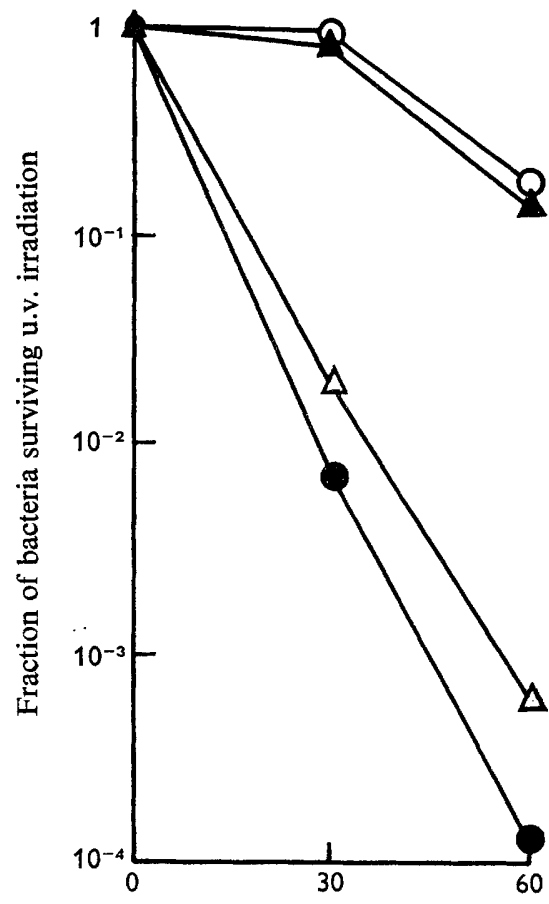

Time of exposure (sec.)

Fig. 2

Fig. I. Dose/log-survival curves for ultraviolet-irradiated Salmonella typhimurium LT $2_{\text {SL675 }}$

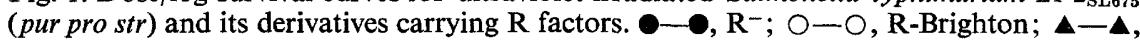
R-Munich; $\triangle-\triangle, \mathbf{R}$-Singapore. Three other $f^{-}$factors gave curves similar to that for R-Brighton. Four other $f^{+}$factors gave curves similar to that shown for R-Singapore.

Fig. 2. Dose/log-survival curves for u.v.-irradiated Salmonella typhimurium LT $2_{\text {SL748 }}$ (met $\operatorname{trp}$ str $r f b$ ) and its derivatives given resistance traits by contact with transductants carrying either some or all of the resistance traits of $\mathrm{R}_{-B}$ Brighton. - $-\mathrm{R}^{-} ; \mathrm{O}-\mathrm{O}, \mathrm{R}^{+}(\mathrm{Tc}, \mathrm{Sm}, \mathrm{Su}$, $\mathrm{Pn}) ; \Delta-\Delta, \mathrm{R}^{+}(\mathrm{Sm}, \mathrm{Su}, \mathrm{Pn}) ; \Delta-\Delta, \mathrm{R}^{+}(\mathrm{Pn})$.

streptomycin-resistance, undetermined because the LT 2 strain used (SL 674) was already streptomycin-resistant. At least two clones were tested for each $\mathrm{R}$ factor, always with consistent results (Fig. I). All the four $f^{-}$factors greatly decreased the susceptibility of strain SL 675 (LT 2 pur pro str) to killing by u.v. radiation. Of the $\mathrm{fi}^{+}$factors tested, R-Munich protected but the other four did not. The five factors which conferred u.v. resistance gave similar protection, about equal to that given by a coll factor; for instance, the survival was between 0.15 and 0.45 after 60 sec. 
exposure, a dose which permitted survival of only about $3 \times 10^{-4}$ of the $\mathrm{R}^{-}$strain. The data though scanty show that all the protecting factors caused the appearance of an initial shoulder in the dose/log-survival curves. Two clones which had acquired only $\mathrm{Tc}$ and $\mathrm{Tc}, \mathrm{Su}$ from R-Munich $\left(f^{+}, \mathrm{Tc}, \mathrm{Sm}, \mathrm{Su}, \mathrm{Cm}, \mathrm{Km}\right.$ ) by conjugation were tested. Both showed the protecting effect. Segregation of the u.v.-protecting character was seen amongst three LT 2 lines given some or all of the resistance traits of R-Brighton ( $f^{-}, \mathrm{Tc}, \mathrm{Sm}, \mathrm{Su}, \mathrm{Pn}$ ) by contact with transductants which had also acquired the ability to conjugate. A clone with only Pn was not protected, whereas one with all four traits and another with all except Tc had the same u.v. resistance as clones given the complete $\mathrm{R}$ factor by conjugation (Fig. 2).

\section{DISCUSSION}

The four $f^{-} \mathrm{R}$ factors examined differed from previously described $\mathrm{R}$ factors in two important respects. First, nearly all transductants which acquired tetracycline resistance also acquired all or most of the other resistance traits of the factor concerned, instead of acquiring only tetracycline resistance. Secondly, about $10 \%$ of the transductant clones tested were able to transmit their transduced resistance traits during growth in mixed culture, instead of none or very few having this ability (Watanabe \& Fukusawa, 1961; Harada et al. 1963). A minority of the transductants which were unable to transmit their traits by conjugation acquired this ability after they were given the plasmid $\mathrm{F}^{\prime}$-I 3 lac (see Harada et al. 1964). We cannot explain these different kinds of behaviour during transduction by phage P 22. Perhaps in these factors, though not in others, the gene for tetracycline resistance is so closely linked to the other drug-resistance genes that a DNA molecule of the length which can be contained within a $\mathbf{P} 22$ phage coat is likely to encompass all the drug-resistance genes of the factor. Those transductants able to transmit all the resistance traits of the factor by conjugation presumably receive the whole of the DNA comprising the $\mathrm{R}$ factor, and this therefore must also be capable of enclosure within a single $\mathbf{P} 22$ phage coat. It is therefore perhaps surprising that only a minority of transductants receive the ability to conjugate instead of all or nearly all, as when $\mathrm{R}$ factors are transduced by the larger phage $\mathrm{P}_{\mathrm{I}}$ in $E$. coli. The four R factors with which this occurred were also the only $\mathrm{f}^{-}$-factors amongst those we examined; but it is possible that they may have a recent common origin since the strains from which they were obtained were all Salmonella typhimurium of phage type $\mathrm{I}$ a, isolated in Great Britain or Holland at about the same time.

The $f^{+}$factors which conferred more than one resistance trait fell into two groups. With three factors the pattern of the transduction was as previously reported for other factors in Salmonella; namely tetracycline resistance was never co-transduced with the other traits, which usually travelled together; and none of the transductants could transmit by conjugation, even after acquisition of $\mathrm{F}^{\prime}-\mathrm{I} 3$ lac. The determinant for the kanamycin (and neomycin) trait of the $\mathrm{R}$ factor isolated by Lebek (1963) behaved as though closely linked with the $\mathrm{Sm}, \mathrm{Su}$ and $\mathrm{Cm}$ determinants, in confirmation of the report of Watanabe et al. (1964b). The other group comprised two $f^{+}$factors which conferred resistance to benzylpenicillin (and cephalosporins) in addition to the Tc $\mathrm{Sm}, \mathrm{Su}, \mathrm{Cm}$ combination. The pattern of transduction of these four determinants was the same as that in other $f^{+}$factors-except that a few transductants after acquisition 
of $F^{\prime}-\mathrm{I} 3$ lac could transmit by conjugation. The benzylpenicillin resistance trait of these two factors, unlike that of the $f^{-}$factors and unlike the kanamycin-resistance trait of the $f^{+}$R-Munich, was never co-transduced with any other trait, though it was transduced by itself at low frequency. Thus, transduction of these factors by phage P 22 produces, not two, but three common transductant classes, none of them able to transmit by conjugation. This may indicate that their DNA is longer than that of previously reported $f^{+}$factors-or it may be that their benzylpenicillin-resistance gene is located in a region of the $\mathrm{R}$-factor linkage group which in other $f^{+}$factors does not cause any obvious alteration of host phenotype, so that its acquisition by transductants would not have been detected.

We easily obtained a strain carrying both R-Munich, an $f^{+}$factor, and R-Brighton, an $f^{-}$factor-as was to be expected since $f i^{+}$and $f^{-}$factors do not exclude each other (Watanabe et al. 1964a). The strain carrying both factors was resistant to six different agents, but each of the numerous classes of transductant obtained could be accounted for by transduction of a fragment either of R-Munich or of R-Brighton. Transductants selected as tetracycline-resistant were of two sorts: large colonies, resistant to no other agent, attributable to transduction of the Tc determinant of R-Munich; and smaller colonies, with several other resistance traits, including the Pn of R-Brighton. This observation confirms the inference of Watanabe et al. (I964a) that a bacterial host can support the continued presence of both an $\mathrm{fi}^{+}$and an $\mathrm{fi}^{-}$factor, without these factors undergoing recombination. The Tc, $\mathrm{Sm}, \mathrm{Su}, \mathrm{Cm}, \mathrm{Km}$ traits of the LT 2 lines obtained by growing a strain carrying an $f^{+}$factor of type Tc, Sm, Su, Cm, with a strain carrying the $f^{+}$factor, R-Barnet, of character $\mathrm{Km}$ might have resulted in recombination of the two $f^{+}$factors (Watanabe et al. 1964a). However, though the $\mathrm{Km}$ trait of the $f^{+}$ R-Munich factor was usually co-transduced with its $\mathrm{Sm}, \mathrm{Su}$ and $\mathrm{Cm}$ traits, the $\mathrm{Km}$ trait and the $\mathrm{Sm}, \mathrm{Su}, \mathrm{Cm}$ group of the strains obtained by mixed growth were never cotransduced (Table 5). This suggests that the strains carried both the parental $f^{+}$factors, rather than a single recombinant factor.

Some of the $\mathrm{R}$ factors protected Salmonella typhimurium strain LT 2 against the bactericidal effect of ultraviolet irradiation to about the same effect as do most colla and most collb factors (Howarth, 1965; Howarth-Thompson, 1966), whereas other factors had little or no effect on the dose/log-survival curves (Fig. r). The mechanism of this protection is unknown. Since such plasmids replicate without being incorporated into the chromosome of their host they presumably carry genes which specify enzymes involved in DNA synthesis and perhaps also in DNA repair, and such enzymes might assist in the restoration of u.v.-damaged DNA. All the $f^{-}$factors we tested protected, as also did one of the five $f^{+}$factors; but some $f^{-}$factors do not protect (A. Siccardi, personal communication). The u.v.-protection effect of $\mathrm{R}$-Brighton appeared to segregate during transduction (Fig .2), so this property may prove a useful new marker for the linkage map of some $\mathrm{R}$ factors.

The $f^{-}$R-Brighton and the $f^{+}$R-Singapore and R-Zambia factors all caused production of $\beta$-lactamase for both benzylpenicillin and cephalothin; Datta \& Kontomichalou (1965) reported such activity for three $\mathrm{R}$ factors, including $\mathrm{R} 18 \mathrm{I}$, which is the factor we term R-Brighton. In their experiments the presence of R-Brighton in either of two strains of Escherichia coli, though it conferred considerable ability to destroy both cephaloridine and 6-aminopenicillanic acid, caused only a twofold increase in the minimal inhibitory concentrations of these drugs; whereas we found 
the same factor in Salmonella typhimurium increased the minimal inhibitory concentration of cephaloridine from $\mathrm{I} \cdot 25$ to $35 \mu \mathrm{g}$. $/ \mathrm{ml}$. (Table 2) and caused a considerable increase in resistance to 6-aminopenicillanic acid. This difference may result from the different hosts or from the different methods of measuring bacterial sensitivity. The determinants of benzylpenicillin resistance (presumably the structural genes for penicillinases) in our $f^{-}$factors differ genetically from those in our $f^{+}$factors in that they are co-transducible with the Sm and Su determinants. It will be interesting to see whether the Pn genes of $f^{-}$and $f^{+}$factors, which presumably arose independently. specify chemically different penicillinases. Datta \& Kontomichalou (I965) found that the substrate profile of the enzyme determined by R I8I8 (= R-Brighton) was very different from that of the enzymes determined by two other $\mathrm{R}$ factors.

We thank Dr N. Datta and Dr K.P. Carpenter for supplying us with strains carrying R factors. W. T. D. is indebted to the Medical Research Council for a Postdoctoral Research Fellowship.

\section{REFERENCES}

Anderson, E. S. \& Datta, N. (1965). Resistance to penicillins and its transfer in Enterobacteriaceae Lancet i, 407 .

Carpenter, K. P. \& Drabble, W. T. (1965). Transferable antibiotic resistance. Br. med. J. ii, 1553.

Datta, N. \& Kontomichalou, P. (1965). Penicillinase synthesis controlled by infectious $R$ factors in Enterobacteriaceae. Nature, Lond. 208, 239.

Datta, N., Lawn, A. M. \& Meynell, E. (I966). The relationship of F type piliation and F phage sensitivity to drug resistance transfer in $\mathrm{R}^{+} \mathrm{F}^{-}$Escherichia coli $\mathrm{K}$ 12. J. gen. Microbiol. 45, 365 .

Davis, B. D. \& Mingroli, E. S. (1950). Mutants of Escherichia coli requiring methionine or vitamin B 12. J. Bact. 6o, I7.

Drabble, W. T. \& Stocker, B. A. D. (I966). Transducibility and other properties of some R (transmissible drug resistance) factors. Heredity, Lond. 21, 166.

Falkow, S., Citarella, R. V., Wohlhieter, J. A. \& Watanabe, T. (1966). The molecular nature of R-factors. J. molec. Biol. 17, 102.

Foley, J. M. \& Perret, C. J. (1962). Screening of bacterial colonies for penicillinase production. Nature, Lond. 195, 287.

Gemski, P. \& Stocker, B. A. D. (1967). Transduction by bacteriophage P 22 in non-smooth mutants of Salmonella typhimurium. J. Bact. $93, \mathrm{I} 588$.

Harada, K., Kameda, M., Suzuki, M. \& Mitsuhashi, S. (1963). Transduction of transmissible drugresistance (R) factors with phage epsilon. J. Bact. 86, 1332.

Harada, K., Kameda, M., Suzuki, M. \& Mitsuhashi, S. (1964). Acquisition of transferability of nontransmissible $\mathrm{R}(\mathrm{Tc})$ factor in co-operation with $\mathrm{F}$ factor and formation of $\mathrm{FR}(\mathrm{Tc})$. J. Bact. $88,1257$.

HowARTH, S. (1965). Resistance to the bactericidal effect of ultraviolet radiation conferred on Enterobacteria by the colicine factor coll. J. gen. Microbiol. $\mathbf{4 0}, 43$.

HowARTH-THOMPSON, S. (I966). Influence of various coll factors on the lethal and mutagenic effects of ultraviolet radiation. Proc. Univ. Otago med. Sch. 44, 5.

LEBEK, G. (1963). Über die Entstehung mehrfachresistenter Salmonellen. Ein experimenteller Beitrag. Zentbl. Bakt. ParasitKde. (Abt I Orig.), 188, 494.

LOEB, T. (I960). Isolation of a bacteriophage specific for $\mathrm{F}^{+}$and $\mathrm{Hfr}$ mating types of Escherichia coli K-12. Science, N.Y. r31, 932.

Meynell, E. \& DatTa, N. (I965). Functional homology of the sex-factor and resistance transfer factors. Nature, Lond. 207, 884 .

MitsuHASHI, S. (1965). Transmissible drug-resistance factor R. Gunma J. med. Sci, 13, I69.

Watanabe, T. (I963). Infective heredity of multiple drug resistance in bacteria. Bact. Rev. 27, 87.

Watanabe, T. \& Fukusawa, T. (I96I). Transduction of resistance factors. J. Bact. 82, 202.

Watanabe, T., Ogata, C. \& Sato, S. (1964b). Six-drug-resistance R factor. J. Bact. 88, 922.

Watanabe, T., Nishida, H., Ogata, C., ARai, T. \& Sato, S. (1964a). Two types of naturally occurring R factors. J. Bact. 88, 716. 